University of Texas at El Paso

ScholarWorks@UTEP

$1-2002$

\title{
Non-Destructive Testing of Aerospace Structures: Granularity and Data Mining Approach
}

\author{
Roberto Osegueda \\ Vladik Kreinovich \\ The University of Texas at El Paso, vladik@utep.edu \\ Lakshmi Potluri \\ Richard Alo
}

Follow this and additional works at: https://scholarworks.utep.edu/cs_techrep

Part of the Computer Engineering Commons

Comments:

UTEP-CS-02-04.

Published in Proceedings of FUZZ-IEEE'2002, Honolulu, Hawaii, May 12-17, 2002, Vol. 1, pp. 685-689.

\section{Recommended Citation}

Osegueda, Roberto; Kreinovich, Vladik; Potluri, Lakshmi; and Alo, Richard, "Non-Destructive Testing of Aerospace Structures: Granularity and Data Mining Approach" (2002). Departmental Technical Reports (CS). 332.

https://scholarworks.utep.edu/cs_techrep/332

This Article is brought to you for free and open access by the Computer Science at ScholarWorks@UTEP. It has been accepted for inclusion in Departmental Technical Reports (CS) by an authorized administrator of ScholarWorks@UTEP. For more information, please contact Iweber@utep.edu. 


\title{
Non-Destructive Testing of Aerospace Structures: Granularity and Data Mining Approach
}

\author{
Roberto Osegueda ${ }^{1}$, Vladik Kreinovich ${ }^{1}$, Lakshmi Potluri ${ }^{1}$, and Richard Aló ${ }^{2}$ \\ ${ }^{1}$ FAST Center, University of Texas at El Paso \\ 500 W. University, El Paso, Texas 79968, USA \\ ${ }^{2}$ University of Houston-Downtown, Houston, Texas 77002 \\ contact email vladik@cs.utep.edu
}

\begin{abstract}
For large aerospace structures, it is extremely important to detect faults, and nondestructive testing is the only practical way to do it. Based on measurements of ultrasonic waves, Eddy currents, magnetic resonance, etc., we reconstruct the locations of the faults. The best (most efficient) known statistical methods for fault reconstruction are not perfect. We show that the use of expert knowledge-based granulation improves the quality of fault reconstruction.
\end{abstract}

\section{INTRODUCTION}

\section{A. Formulation of the Problem}

For large aerospace structures, it is extremely important to detect faults, and non-destructive testing is the only practical way to do it. Based on measurements of ultrasonic waves, Eddy currents, magnetic resonance, etc., we reconstruct the locations of the faults.

The best (most efficient) known statistical methods for such reconstruction use our knowledge of physics and the known statistical characteristics of noise and measurement errors. We describe these methods in Section II. These methods are efficient but not perfect: they miss some fault locations and erroneously mark some non-faulty places as potential faults.

\section{B. What We Are Planning to Do}

A natural statistical way to improve the quality of fault detection is to use more adequate statistical techniques, e.g., to take into consideration correlation between different measurements (which becomes more and more important as the number of measurements increases) and/or use better statistical decision making techniques.

However, as we show in Section III, if we simply use new techniques, the quality of fault detection often deteriorates instead of improving. The reason is that when we apply statistical techniques, we need to either treat the whole plate as a sample, or subdivide it into zones, and the quality of fault detection depends on the adequacy of this granulation. To improve the efficiency of fault detection, we must therefore maximally use the expert knowledge and data mining to get a better granulation. We show that this indeed leads to an improved quality of fault detection.
In Section IV, we show that there is an additional problem with simply using sophisticated statistical methods. The problem is that when we move to sophisticated statistical models, the corresponding computations often become computationally intractable (NP-hard), so we need expert knowledge to solve these problems.

\section{BEST EXISTING STATISTICAL ALGORITHM FOR FAULT DETECTION AND ITS DRAWBACKS}

\section{A. Main Idea}

Let us first describe the existing technique [8,9]. We start with the results $x_{i}(A)$ of measuring $n$ different quantities at different points $A$. The main idea behind this method is as follows: Based on the measured values, we estimate the corresponding probability distribution; points for which the corresponding measurement results $x_{1}(A), \ldots, x_{n}(A)$ are highly improbable (i.e., for which the value of the corresponding probability density $\rho$ is below a certain threshold $\rho_{0}$ ) are most likely faults.

\section{B. Main Idea in More Detail}

If all the measurements are independent and normally distributed with average $a_{i}$ and standard deviation $\sigma_{i}$, then the probability density $\rho\left(x_{1}(A), \ldots, x_{n}(A)\right)$ is proportional to $\exp \left(-\frac{1}{2} \cdot \sum_{i=1}^{n} z_{i}(A)^{2}\right)$, where

$$
z_{i}(A) \stackrel{\text { def }}{=} \frac{x_{i}(A)-a_{i}}{\sigma_{i}}
$$

is a normalized result of $i$-th measurement at the point $A$. In this case, the fault-detecting inequality $\rho \leq \rho_{0}$ is equivalent to the inequality $\sum_{i=1}^{n} z_{i}(A)^{2} \geq c_{0}$ for some constant $c_{0}$. In engineering, it is customary to use $2 \sigma$ rule, which corresponds to $5 \%$ error. In this case, we select the value of $c_{0}$ for which, for the ideal Gaussian distribution, exactly $5 \%$ of points are classified as faults.

\section{How to Compute $a_{i}$ and $\sigma_{i}$ ?}

In order to transform this idea into an algorithm, we must first decide on how to estimate $a_{i}$ and $\sigma_{i}$. If there are no faults, and all the measurements are normally distributed, then we can estimate $a_{i}$ and $\sigma_{i}$ by using the standard 
statistical formulas:

$$
a_{i}=\frac{1}{N} \cdot \sum_{A} x_{i}(A) ; \quad \sigma_{i}=\sqrt{\frac{1}{N-1} \cdot \sum_{A}\left(x_{i}(A)-a_{i}\right)^{2}},
$$

where $N$ is the total number of measurements. However, in real-life situations, when there are outliers, these outliers will affect the results of computing $a_{i}$ and $\sigma_{i}$. To eliminate this effect and use only non-outlier points in computing $a_{i}$ and $\sigma_{i}$, the authors of $[8,9]$ propose to use the following iterative techniques often used in data mining:

- first, we compute the values $a_{i}$ and $\sigma_{i}$ based on all the measurements;

- then, we mark the points $A$ for which $\left|x_{i}(A)-a_{i}\right|>$ $2 \sigma_{i}$ as suspicious, and re-compute the values $a_{i}$ and $\sigma_{i}$ based only on un-marked points $A$;

- for the new values of $a_{i}$ and $\sigma_{i}$, we again mark all the points for which $\left|x_{i}(A)-a_{i}\right|>2 \sigma_{i}$ (i.e., which are outside the $2 \sigma$ interval $\left.\left[a_{i}-2 \sigma_{i}, a_{i}+2 \sigma_{i}\right]\right)$, and re-compute $a_{i}$ and $\sigma_{i}$ using only the remaining unmarked points, etc.

This procedure continues until the values $a_{i}$ and $\sigma_{i}$ stop changing.

\section{B. The Need for Granulation}

If we simply apply the above idea with thus computed $a_{i}$ and $\sigma_{i}$, we do not get very good fault detection results: it detects all points in the interior of the tested plate, but its results on the edges are not that good. The reason why the method does not work well on edges is that the above method assumes that the probabilities are the same in all the points $A$, while on the edges, the physical characteristics are different and therefore, the corresponding probabilities are also different.

It is therefore reasonable to granulate the tested plate, i.e., to divide it into zones which more or less similar physical characteristics. Since the main reason for the difference between physical characteristics at different points is due to different closeness of these points to the edge, it is reasonable to divide the plate into zones by dividing the interval of possible distance to the edge into sub-intervals $\left[0, d_{1}\right],\left[d_{1}, d_{2}\right]$, etc., so that points whose distance to the edge is smaller than $d_{1}$ are assigned to one zone, points whose distance is from $d_{1}$ to $d_{2}$ are assigned to the second zone, etc. Then, within each zone, we only declare a point to be a fault if it is an outlier when compared to the averages and standard deviations measured by only using similar points, i.e., measured only by using points within this zone.

\section{Final Detail: Separating Actual Faults from Sensor Malfunctions}

The algorithm is almost ready, the only remaining detail is that some individual outlying measurements $x_{i}(A)$ can be caused not by faults, but by a malfunction of the corresponding sensors. A typical difference between such points and actual faults is that actual faults are usually continuous, they contain not just a single point $A$, but also several points close to $A$. Thus, if within a certain neighborhood $\{A, B, \ldots\}$ of a point $A$, only one type of measurement $x_{i}(A), x_{i}(B), \ldots$ indicates a fault (i.e., for only this type of measurement, $x_{i}(B)$ is outside the "two sigma" interval $\left.\left[a_{i}-2 \sigma_{i}, a_{i}+2 \sigma_{i}\right]\right)$, we dismiss the outlying value $x_{i}(A)$ as a possible malfunction, and only use the remaining values in our statistical analysis.

\section{Main Drawback: The Methods Should Be Further Improved}

In $[8,9]$, the above method was tested on the example of an $11^{\prime \prime} \times 11^{\prime \prime}$ plate from an actual airplane (B-52) with 16 artificially added faults: 8 squares and 8 circles. Of these 16 faults, 8 are inside the plate: four squares of sides $1 / 2^{\prime \prime}$, $3 / 8^{\prime \prime}, 1 / 4^{\prime \prime}$, and $1 / 8^{\prime \prime}$; and four circles of diameters $1 / 2^{\prime \prime}$, $3 / 8^{\prime \prime}, 1 / 4^{\prime \prime}$, and $1 / 8^{\prime \prime}$; and 8 are of the exact same size but on the edge of the plate: four squares of sides $1 / 2^{\prime \prime}, 3 / 8^{\prime \prime}$, $1 / 4^{\prime \prime}$, and $1 / 8^{\prime \prime}$; and four circles of diameters $1 / 2^{\prime \prime}, 3 / 8^{\prime \prime}$, $1 / 4^{\prime \prime}$, and $1 / 8^{\prime \prime}$. For this plate, we have 7 different measurements: two measurements of Pulse Echo corresponding to different frequency, measurement of Eddy current, and four measurements of magnetic resonance corresponding to four different frequencies.

The conclusion is that fault detection provided by the statistical method described in Section II is good but not perfect.

\section{THE NEW ALGORITHM: MOTIVATION, DESCRIPTION, AND RESULTS}

\section{A. Main Idea: Using Correlation}

To get a better fault detection, we can look into the assumption that we made. One such assumption is the statistical independence of different measurements.

Our statistical analysis shows that although some pairs of measurements are indeed almost independent, there is is some correlation between some other measurement results. For example, there is a sizable correlation between two measurements of resonance corresponding to different frequencies: the corresponding correlation coefficient is $\approx 54 \%$.

For correlated normally distributed random variables, the probability is a function not of $\sum_{i=1}^{n} z_{i}(A)^{2}$, but of a more general quadratic form $\sum_{i=1}^{n} \sum_{j=1}^{n} b_{i j} \cdot z_{i}(A) \cdot z_{j}(A)$, where the matrix $b_{i j}$ is the inverse to the covariance matrix $C_{i j}$ that describes the correlation between the measurements. Each element $C_{i j}$ of this matrix is the average of the product $z_{i}(A) \cdot z_{j}(A)$ over different points $A$. It is therefore reasonable to do the following: 
- compute the elements of the covariance matrix $C_{i j}$;

- invert this matrix getting $b_{i j}$; and then

- use the expression $\sum_{i=1}^{n} \sum_{j=1}^{n} b_{i j} \cdot z_{i} \cdot z_{j}$ instead of the expression $\sum_{i=1}^{n} z_{i}^{2}$ to detect the faults.

\section{B. This Idea Is Not Sufficient, Additional Expert Knowledge-Based Granulation Is Needed}

The idea sounds reasonable, however, when we first applied it, the quality of fault detection deteriorated instead of improving. The most troubling deterioration was the increase in the number of false negatives, i.e., undetected fault points. In the original method, we had 11 false negatives, but with our application, we had 20 false negatives - almost twice as many. Why?

The reason is that when we apply statistical techniques, we need to either treat the whole plate as a sample, or subdivide it into zones, and the quality of fault detection depends on the adequacy of this granulation. To improve the efficiency of fault detection, we must therefore maximally use the expert knowledge and data mining to get a better granulation.

It is reasonable to expect that expert knowledge can help. It is well known that methods based on expert knowledge (especially methods using data fusion) lead to reasonably good algorithms for fault detection; see, e.g., $[2,5,7,10,17]$. It is also known that the use of expert knowledge can improve statistical techniques of fault detection; see, e.g., [1, 5, 12].

Based on the expert knowledge, we decided to divide the plate into two zones:

- the interior of the plate, i.e., all the points which are at least $3 / 4^{\prime \prime}$ away from the edge; and

- the remaining points - which are thus close to the edge.

We performed our statistical analysis separately on these two zones. The interior zone contains sufficiently many points to lead to a statistically significant non-zero correlation between some measurements. In contrast, the edge zone contains too few points, not enough to detect any statistically significant correlation between the measurements. Therefore, for this zone, we used the covariances computed based on the interior zone.

When we took these covariances into consideration in the above formulas, we ended up with the decreased number of false negatives (9 instead of the original 11). We also got a decrease in the overall number of false positives (i.e., points erroneously marked as faults) from the original 5,986 to 5,842 . In other words, the use of expert knowledge in granulation indeed leads to an improved quality of fault detection.

\section{TOWARDS FURTHER IMPROVEMENTS: ANALYZING COMPUTATIONAL COMPLEXITY OF COMPUTING COVARIANCE}

\section{A. Main Idea}

The new algorithm proposed in Section III produces an improvement in the detection of outliers in comparison with the best existing algorithm, but this improvement is slight. In order to get better results, a natural idea is to use an even more realistic description of the problem. One thing that is not realistic in the current description is that in computing the covariance we assume that the measurement results are exact.

In real life, measurements are never $100 \%$ accurate. The result $\widetilde{x}$ of measuring a physical quantity $x$ (e.g., temperature) may differ from the actual value of that quantity. E.g., if you have weighed yourself, and the result is 125 pounds, this does not mean that your weight equals exactly 125 . If the scales have an accuracy \pm 2 , then the actual weight can be any number from $123(=125-2)$ to $127(=125+2)$.

So the data that we process are not absolutely precise. This inaccuracy leads to the inaccuracy in the result of data processing, in our case, in the estimated values of covariance. The problem is to estimate the resulting inaccuracy.

In many cases, the manufacturer of a measuring instrument provides us with the probabilities of different values of a measurement error. For such cases, there exist numerous methods that compute statistical characteristics of the resulting error (see, e.g., $[3,13]$ ).

In many other cases, however, the values of the probabilities are not known. Instead, the manufacturer provides us with the guaranteed accuracy $\Delta$, i.e., with a guaranteed upper bound of the error $\Delta x=\widetilde{x}-x$ (e.g., "error cannot exceed 0.1"). If our measurement results is $\widetilde{x}$, then the possible values of $x=\widetilde{x}-\Delta x$ form an interval $\left[x^{-}, x^{+}\right]$, where $x^{-}=\widetilde{x}-\Delta$ and $x^{+}=\widetilde{x}+\Delta$.

Taking interval uncertainty into consideration does lead to improved fault detection results in non-destructive testing; see, e.g., $[14,15,16]$. It is therefore reasonable to take interval uncertainty into consideration in our correlation technique as well.

In the standard computation of covariance, we start with the values $x_{1}, \ldots, x_{n}, y_{1}, \ldots, y_{n}$, and we compute the covariance as

$$
C=\frac{1}{n} \sum_{i=1}^{n}\left(x_{i}-\bar{x}\right) \cdot\left(y_{i}-\bar{y}\right),
$$

where

$$
\bar{x}=\frac{1}{n} \sum_{i=1}^{n} x_{i} ; \quad \bar{y}=\frac{1}{n} \sum_{i=1}^{n} y_{i} .
$$

If we take interval uncertainty into consideration, then, after each measurement, we do not get the exact values 
of $x_{1}, \ldots, x_{n}, y_{1}, \ldots, y_{n}$; instead, we only have intervals $\left[x_{1}^{-}, x_{1}^{+}\right], \ldots,\left[x_{n}^{-}, x_{n}^{+}\right],\left[y_{1}^{-}, y_{1}^{+}\right], \ldots,\left[y_{n}^{-}, y_{n}^{+}\right]$. Depending on what are the actual values of $x_{1}, \ldots, x_{n}, y_{1}, \ldots, y_{n}$ within these intervals, we get different values of covariance. To take the interval uncertainty into consideration in our covariance techniques, we need to be able to describe the interval $\left[C^{-}, C^{+}\right]$of possible values of covariance $C$.

So, we arrive at the following problems: given the intervals $\left[x_{i}^{-}, x_{i}^{+}\right],\left[y_{i}^{-}, y_{i}^{+}\right]$, compute the lower and upper bounds $C^{-}$and $C^{+}$for the interval of possible values of covariance.

\section{B. To Implement the Main Idea, We Need Expert Knowledge: Theorems}

It turns out that these problems are computationally difficult, or, in precise terms, NP-hard (for exact definitions of NP-hardness, see, e.g., $[4,6,11])$.

Theorem 1. The problem of computing $C^{+}$from the interval inputs $\left[x_{i}^{-}, x_{i}^{+}\right],\left[y_{i}^{-}, y_{i}^{+}\right]$is NP-hard.

Theorem 2. The problem of computing $C^{-}$from the interval inputs $\left[x_{i}^{-}, x_{i}^{+}\right],\left[y_{i}^{-}, y_{i}^{+}\right]$is NP-hard.

These results are not unexpected: many easy computational problems (e.g., solving systems of linear equations) become NP-hard if we take interval uncertainty into consideration; see, e.g., [6].

What does this computational hardness means in practical terms? It means that if we only use the measurement results - and do not use any knowledge of experts, then the corresponding computations are difficult. Thus, to solve the corresponding practical problems, we must use expert knowledge in addition to measurement results.

\section{Proof of Theorem 1}

By definition, a problem is NP-hard if any problem from the class NP can be reduced to it. Therefore, to prove that a problem $\mathcal{P}$ is NP-hard, it is sufficient to reduce one of the known NP-hard problems $\mathcal{P}_{0}$ to $\mathcal{P}$. In this case, since $\mathcal{P}_{0}$ is known to be NP-hard, this means that every problem from the class NP can be reduced to $\mathcal{P}_{0}$, and since $\mathcal{P}_{0}$ can be reduced to $\mathcal{P}$, thus, the original problem from the class NP is reducible to $\mathcal{P}$.

For our proof, as the known NP-hard problem $\mathcal{P}_{0}$, we take a subset problem: given $n$ positive integers $s_{1}, \ldots, s_{n}$, to check whether there exist signs $\varepsilon_{i} \in\{-1,+1\}$ for which the signed sum $\sum_{i=1}^{n} \varepsilon_{i} \cdot s_{i}$ equals 0 .

We will show that this problem can be reduced to the problem of computing $C^{+}$, i.e., that to every instance $\left(s_{1}, \ldots, s_{n}\right)$ of the problem $\mathcal{P}_{0}$, we can put into correspondence such an instance of the $C^{+}$-computing problem that based on its solution, we can easily check whether the desired signs exist.

As this instance, we take the instance corresponding to the intervals $\left[x_{i}^{-}, x_{i}^{+}\right]=\left[y_{i}^{-}, y_{i}^{+}\right]=\left[-s_{i}, s_{i}\right]$. We want to to show that for the corresponding problem, $C^{+}=C_{0}$, where we denoted

$$
C_{0} \stackrel{\text { def }}{=} \frac{1}{n} \cdot \sum_{i=1}^{n} s_{i}^{2}
$$

if and only if there exist signs $\varepsilon_{i}$ for which $\sum \varepsilon_{i} \cdot s_{i}=0$.

To prove this, let us first show that in all cases, $C^{+} \leq$ $C_{0}$. Indeed, it is known that the covariance $C$ is bounded by the product $\sigma_{x} \cdot \sigma_{y}$ of standard deviations of $x$ and $y$. By definition of a standard deviation,

$$
\sigma_{x}^{2}=\frac{1}{n} \cdot \sum_{i=1}^{n} x_{i}^{2}-(\bar{x})^{2} .
$$

Since $x_{i} \in\left[-s_{i}, s_{i}\right]$, we can conclude that $x_{i}^{2} \leq s_{i}^{2}$; since $(\bar{x})^{2} \geq 0$, we thus conclude that

$$
\sigma_{x}^{2} \leq \frac{1}{n} \cdot \sum s_{i}^{2}=C_{0} .
$$

So, $\sigma_{x} \leq \sqrt{C_{0}}$; similarly, $\sigma_{y} \leq \sqrt{C_{0}}$, hence $C \leq \sigma_{x} \cdot \sigma_{y} \leq$ $\sqrt{C_{0}} \cdot \sqrt{C_{0}}=C_{0}$. In other words, every possible value $C$ of the covariance is smaller than or equal to $C_{0}$. Thus, the largest of these possible values, i.e., $C^{+}$, also cannot exceed $C_{0}$, i.e., $C^{+} \leq C_{0}$.

Let us now show that if $C^{+}=C_{0}$, then the desired signs exist. Indeed, if $C^{+}=C$, this means that for the corresponding values of $x_{i}$ and $y_{i}$, the covariance $C$ is equal to $C_{0}$, i.e.,

$$
C=C_{0}=\frac{1}{n} \cdot \sum_{i=1}^{n} s_{i}^{2} .
$$

On the other hand, we have shown that in all cases (and in this case in particular), $C \leq \sigma_{x} \cdot \sigma_{y} \leq \sqrt{C_{0}} \cdot \sqrt{C_{0}}=C_{0}$. If $\sigma_{x}<\sqrt{C_{0}}$, then we would have $C<C_{0}$. So, if $C=C_{0}$, we have $\sigma_{x}=\sigma_{y}=\sqrt{C_{0}}$, i.e., $\sigma_{x}^{2}=\sigma_{y}^{2}=C_{0}$. We know that $x_{i}^{2} \leq s_{i}^{2}$; since $(\bar{x})^{2} \geq 0$ hence $\sigma_{x}^{2} \leq C_{0}$. If $\left|x_{i}\right|^{2}<s_{i}^{2}$ or $(\bar{x})^{2}>0$, then we would have $\sigma_{x}^{2}<\bar{C}_{0}$. Thus, the only way to have $\sigma_{x}^{2}=C_{0}$ is to have $x_{i}^{2}=s_{i}^{2}$ and $\bar{x}=0$. The first equality leads to $x_{i}= \pm s_{i}$, i.e., to $x_{i}=\varepsilon_{i} \cdot s_{i}$ for some $\varepsilon_{i} \in\{-1,+1\}$. The second equality then leads to $\sum_{i=1}^{n} \varepsilon_{i} \cdot s_{i}=0$. So, if $C^{+}=C_{0}$, then the desired signs do exist.

To complete the proof of Theorem 1, we must show that, vice versa, if the desired signs $\varepsilon_{i}$ exist, then $C^{+}=C_{0}$. Indeed, in this case, for $x_{i}=y_{i}=\varepsilon_{i} \cdot s_{i}$, we have $\bar{x}=\bar{y}=0$ and $x_{i} \cdot y_{i}=s_{i}^{2}$, hence

$$
C=\frac{1}{n} \cdot \sum_{i=1}^{n}\left(x_{i}-\bar{x}\right) \cdot\left(y_{i}-\bar{y}\right)=\frac{1}{n} \cdot \sum_{i=1}^{n} s_{i}^{2}=C_{0} .
$$

The theorem is proven. 


\section{Proof of Theorem 2}

This proof is similar, with the only difference that in this case, we use the other part of the inequality $|C| \leq \sigma_{x} \cdot \sigma_{y}$, namely, that $C \geq-\sigma_{x} \cdot \sigma_{y}$, and in the last part of the proof, we take $y_{i}=-x_{i}$.

\section{CONCLUSION}

In this paper, we have shown that granularity based on expert knowledge can drastically improve the quality of fault detection in aerospace structures.

\section{ACKNowledgments}

This work was supported in part by NASA under cooperative agreement NCC5-209 and grant NCC 2-1232, by the Future Aerospace Science and Technology Program (FAST) Center for Structural Integrity of Aerospace Systems, effort sponsored by the Air Force Office of Scientific Research, Air Force Materiel Command, USAF, under grants numbers F49620-95-1-0518 and F49620-00-10365, by grant No. W-00016 from the U.S.-Czech Science and Technology Joint Fund, by NSF grants CDA9522207, ERA-0112968, and 9710940 Mexico/Conacyt, and by IEEE/ACM SC2001 Minority Serving Institutions Participation Grant.

\section{REFERENCES}

[1] C. Ferregut, R. A. Osegueda, Y. Mendoza, V. Kreinovich, and T. J. Ross, "Aircraft Integrity and Reliability", In: J. Booker, J. Parkinson, and T. R. Ross (eds.), Combined Fuzzy Logic and Probability Applications, SIAM, Philadelphia, 2002 (to appear).

[2] C. Ferregut, R. A. Osegueda, and A. Nuñez, A. (eds.), Proceedings of the International Workshop on Intelligent NDE Sciences for Aging and Futuristic Aircraft, El Paso, TX, September 30-October 2, 1997.

[3] W. A. Fuller, Measurement error models, J. Wiley \& Sons, New York, 1987.

[4] M. R. Garey and S. D. Johnson, Computers and Intractability: A Guide to the Theory of NPCompleteness, W.H. Freeman and Company, New York, 1979.

[5] X. E. Gros, NDT Data Fusion, J. Wiley, London, 1997.

[6] V. Kreinovich, A. Lakeyev, J. Rohn, and P. Kahl, Computational complexity and and feasibility of data processing and interval computations Kluwer, Dordrecht, 1997.

[7] M. Krishna, V. Kreinovich, and R. A. Osegueda, "Fuzzy Logic in Non-Destructive Testing of Aerospace Structures", Proceedings of the 1999 IEEE Midwest Symposium on Circuits and Systems, Las Cruces, New Mexico, August 8-11, 1999, Vol. 1, pp. $431-434$.

[8] R. A. Osegueda,S. Seelam,C. Holguin,V. Kreinovich, and C. W. Tao, "Statistical and Dempster-
Shafer Techniques in Testing Structural Integrity of Aerospace Structures", Proc. Int'l Conf. on Intelligent Technologies, Bangkok, Thailand, December 1315, 2000, pp. 383-389.

[9] R. A. Osegueda,S. Seelam,C. Holguin,V. Kreinovich, and C. W. Tao, "Statistical and DempsterShafer Techniques in Testing Structural Integrity of Aerospace Structures", International Journal of Uncertainty, Fuzziness, Knowledge-Based Systems (IJUFKS), 2001, Vol. 9, No. 6 (to appear).

[10] R. A. Osegueda, C. Ferregut, V. Kreinovich, S. Seelam, and H. Schulte, "Fuzzy (Granular) Levels of Quality, With Applications to Data Mining and to Structural Integrity of Aerospace Structures", Proc. NAFIPS'2000, Atlanta, Georgia, July 13-15, 2000, pp. $348-352$.

[11] C. H. Papadimitriou, Computational Complexity, Addison Wesley, San Diego, 1994.

[12] T. J. Ross, C. Ferregut, R. A. Osegueda, and V. Kreinovich, "System Reliability: A Case When Fuzzy Logic Enhances Probability Theory's Ability to Deal With Real-World Problems", Proc. NAFIPS'99, New York City, June 10-12, 1999, pp. $81-84$.

[13] H. M. Wadsworth, Jr. (eds.), Handbook of statistical methods for engineers and scientists, McGraw-Hill Publishing Co., New York, 1990.

[14] K. Worden, R. Osegueda, C. Ferregut, S. Nazarian, D. L. George, M. J. George, V. Kreinovich, "Interval Methods in Non-Destructive Testing of Aerospace Structures and in Mammography", International Conference on Interval Methods and their Application in Global Optimization (INTERVAL'98), April 20-23, Nanjing, China, Abstracts, 1998, pp. 152-154.

[15] K. Worden, R. Osegueda, C. Ferregut, S. Nazarian, E. Rodriguez, D. L. George, M. J. George, V. Kreinovich, O. Kosheleva and S. Cabrera, "Interval Approach to Non-Destructive Testing of Aerospace Structures and to Mammography", In: G. Alefeld and R. A. Trejo (eds.), Interval Computations and its Applications to Reasoning Under Uncertainty, Knowledge Representation, and Control Theory. Proceedings of MEXICON'98, Workshop on Interval Computations, 4th World Congress on Expert Systems, Mexico City, Mexico, 1998.

[16] K. Worden et al., "Interval Methods in NonDestructive Testing of Material Structures", Reliable Computing, 2001, Vol. 7, No. 4, pp. 341-352.

[17] Y. Yam, R. Osegueda and V. Kreinovich, "Towards Faster, Smoother, and More Compact Fuzzy Approximation, with an Application to Non-Destructive Evaluation of Space Shuttle's Structural Integrity", Proc. NAFIPS'99, New York City, June 10-12, 1999, pp. 243-247. 